\title{
Variation of Platelet Indices among patients with Acute Stroke and the need for their routine documentation
}

\author{
Muthuvel Esaki ${ }^{1}$, Anish.S.Bharatwaj ${ }^{2 *}$, Yogalakshmi E ${ }^{2}$ and Architha Ellappalayam ${ }^{3}$ \\ ${ }^{1}$ Saveetha Medical College and Hospital, Thandalam, Tamil Nadu, India \\ ${ }^{2}$ Department of Pathology, Saveetha Medical College and Hospital, Thandalam, Tamil Nadu, India \\ ${ }^{3}$ Wageningen University, Netherlands
}

\begin{abstract}
Background: Acute Stroke is characterized by sudden appearance of focal deficit of brain function most commonly hemiplegia, which may be accompanied by higher centre dysfunction, sensory loss and visual field defects. Based on the pathology, it is classified as Ischemic or Hemorrhagic stroke. In both the conditions, there is an inevitable involvement of platelets. In the former, platelet activation leading to a thrombus formation is the pathogenic feature whereas in the latter, platelet activation to stop the bleeding is seen.

In this study we aim to find the association between Platelet Count, Platelet Indices; Mean Platelet Volume (MPV), Platelet Distribution Width (PDW) and Stroke.

Methods: It was a prospective study done in which 70 consecutive patients were divided into ischemic and hemorrhagic strokes. Proper Exclusion criteria were carried out and the patients' blood samples were taken. Platelet indices were measured with a SYSMEX analyser. Statistical analysis was done using 2 sample T Tests using SPSS software.

Result: The Parameters MPC, MPV and PDW all showed a significant association ( $\mathrm{P}=0.04, \mathrm{P}=0.00, \mathrm{P}=0.00$ respectively) between the stroke patients and control group. Furthermore, a significant difference was found between MPC of patients with ischemic and hemorrhagic stroke $(\mathrm{P}=0.025)$.

Conclusion: Platelet Indices are altered in patients with Stroke when compared to normal patients with no history of CAD or CVD or other lifestyle diseases. Further studies and researches have to be undertaken to see if Platelet Indices are indeed a worthy prognostic tool.
\end{abstract}

Keywords: Platelet Indices, Stroke, Ischemic, Hemorrhagic, Platelets

\section{Introduction}

Platelets constitute one of the three formed elements of blood. They have been known to aid in several physiologic processes, most important being hemostasis..$^{[1]}$ They are fragments of megakaryocyte cytoplasm and are small discoid entities ${ }^{[2]}$ having a diameter of roughly $1-4 \mu \mathrm{m} \cdot{ }^{[3]}$ [4] Their contents include cytoskeleton filaments, alpha and delta granules containing critical components of hemostasis and lysosymes. In any pathologic state that leads to activation of platelets, they assume a change in shape and size. ${ }^{[5]}$ It is well known that larger platelets are capable of increased aggregation and also contain more granules. ${ }^{[6][7][8]}$

There exist parameters to monitor platelets and their changes, namely; Mean Platelet Volume (MPV), Mean Platelet Count (MPC), Plateletcrit (PCT), and Platelet Distribution Width (PDW) [9]. Together, the above mentioned parameters comprise the platelet indices. Studies have shown that these indices are good indicators of thrombotic states. ${ }^{[10]}$ Increased Mean Platelet Volume
(MPV) has been associated with Myocardial Infarction, Transient Ischemic Attacks and Ischemic Stroke. ${ }^{[1][12][13][14]}$

Acute Stroke is characterized by sudden appearance of focal deficit of brain function most commonly hemiplegia, which may be accompanied by higher centre dysfunction, sensory loss and visual field defects. ${ }^{[15][16]}$ Based on the pathology, it is classified as Ischemic or Hemorrhagic stroke. ${ }^{[16]}$ In both the conditions, there is an inevitable involvement of platelets. In the former, activation of platelets leading to a thrombus formation is the pathogenic feature ${ }^{[17]}$ whereas in the latter, platelet activation to stop the bleeding is seen. ${ }^{[18]}$ Studies have strongly associated an increased MPV with Ischemic stroke and the same has been used as a prognostic marker ${ }^{[19]}$ Certain studies also prove that raised MPV is a common finding in recurrent stroke. ${ }^{[2][22]}$

Since the advent of modern laboratory instrumentation, the ability to record platelet indices has been within reach. Notable platforms that record platelet indices include Sysmex and Lanex. But owing to setbacks, most of which are attributed to lack of correlation with disease and no 
proper standardization criteria or reference ranges, ${ }^{[23][24]}$ documentation is seldom done.

\section{Materials and Methods}

This Case Control Study was done for a period of 7 months, from March 2017 to September 2017 after getting an approval from the IEC. During this period, 70 consecutive patients who presented to the General Medicine Outpatient of Saveetha Medical College and Hospital, with clinical features of stroke were enrolled for the study, the exclusion criteria being a history of Auto Immune Diseases, inflammatory conditions, bleeding disorders and history of Anti-Platelet Drug medication. For controls, 100 consecutive persons who had come to the Hospital for the purpose of regular checkup were enrolled for the study, the exclusion criteria being no history of Coronary Artery Disease, Diabetes Mellitus, Dyslipidaemia, Anti Platelet Drug medications, bleeding disorders and Hypertension. Each patient was subjected to detailed history taking and clinical examination for confirmation of Acute Stroke and diagnosis was further confirmed by Computed Tomography Plain and MRI where necessary. The blood samples drawn for hematological investigations were used for the purpose of the study, thereby avoiding exclusive blood withdrawal. The samples were fed into a Sysmexxn-1000 Auto Analyzer. 2 sampled T tests were done using SPSS software.

Limitations of the study include measurement related artifacts and time of collection of blood samples which may potentially affect the findings. Second, we did not measure other physiological parameters like C Reactive Protein and Fibrinogen and vWF levels that can affect platelet activity.

\section{Result}

70 patients with Stroke were included in the study with mean age of $56 \pm 0.62$. Of these, 26 were females and 44 were males. The mean age of controls was $26.16 \pm 6.608$.
When platelet count was measured and compared, the platelet count was $2.74 \pm 1.16 \times 10^{5} / \mathrm{mm}^{3}$ for the test group and $2.44 \pm 0.71 \times 10^{5} / \mathrm{mm}^{3}$ for controls. Upon performing $\mathrm{T}$ test between test group and control group, a significant difference was noticed $(\mathrm{P}=0.042)$. This is shown in Table 1 in concerned section.

The next parameter to be compared was Mean Platelet Volume. The average MPV for the test group was $10.84 \pm 1.08 \mathrm{fL}$ and for the control group was $8.54 \pm 0.76$ $\mathrm{fL}$. A significant difference of 0.000 was found upon performing $\mathrm{T}$ Test. This is shown in Table 2 in concerned section.

The next parameter compared was Platelet Distribution Width. The average PDW for the test group was $12.75 \pm 2.22$ $\mathrm{fL}$ whereas for the control group it was $15.66 \pm .388 \mathrm{fL}$. A P value of .000 was obtained upon performing a T Test. This is shown in the Table 3 in concerned section.

Lastly, we compared the platelet indices for differences between patients with ischemic and hemorrhagic strokes. $\mathrm{T}$ Test was done to compare the values of platelet indices and to look for significant change.

Mean Platelet Count among Hemorrhagic Stroke patients was $1.90 \pm 0.994 \times 10^{5} / \mathrm{mm}^{3}$ and that among Ischemic Stroke patients was $2.81 \pm 0.981 \times 10^{5} / \mathrm{mm}^{3}$. A Significant P value of 0.025 was obtained. This is shown in Table 4 in concerned section. Significant differences however weren't recorded with respect to Mean Platelet Volume and Platelet Distribution Width.

\section{Discussion}

It is proven beyond doubt that Platelets have a major role to play in arterial thrombosis and as such may contribute to the pathogenesis of Arteriosclerotic changes. ${ }^{[20]}$ Based on the baseline platelet activity, considerable insight into their behavior in pathological states can be obtained. In this

Table 1: Comparison of Platelet Count between Test and Control groups

\begin{tabular}{|c|c|c|c|}
\hline Platelet count & Mean & Standard Deviation & P value \\
\cline { 1 - 3 } Test & 2.742 & 1.16 & \multirow{2}{*}{0.042} \\
\hline Control & 2.447 & .71 & \\
\hline
\end{tabular}

Table 2: Comparison of Mean Platelet Volume between Test and Control groups

\begin{tabular}{|c|c|c|c|}
\hline MPV & Mean & Standard Deviation & P Value \\
\hline Test & 10.845 & 1.086 & \multirow{2}{*}{0.000} \\
\hline Control & 8.54 & 0.764 & \\
\hline
\end{tabular}

Table 3: Comparison of Platelet Distribution width between Test and Control groups

\begin{tabular}{|c|c|c|c|}
\hline PDW & Mean & Standard Deviation & P Value \\
\hline Test & 12.75 & 2.22 & \multirow{2}{*}{0.000} \\
\hline Control & 15.66 & 0.38 & \\
\hline
\end{tabular}


Table 4: A Comparison of Platelet Counts between patients with Ischemic and Hemorrhagic stroke

\begin{tabular}{|c|c|c|c|}
\hline Platelet Count & Mean & Standard Deviation & P Value \\
\hline Hemorrhagic & 1.9 & .994 & \multirow{2}{*}{0.025} \\
\hline Ischemic & 2.81 & .981 & \\
\hline
\end{tabular}

study, an attempt has been made to study their changes, through the three indices Mean Platelet Volume, Mean Platelet Count and Platelet Distribution Width, with respect to the pathological basis of a Cerebrovascular Accident.

Having computed the data obtained and after performing statistical tests, the following pertinent observations were made. The MPC was raised in the test group and a significant value of 0.042 was obtained. This was similar to observations made by Waseem F Al-Tameemi et al. ${ }^{[20]}$ This highlights their role in the pathogenesis, ie: Formation of thrombus that causes ischemia and interruption of blood flow to the neurons. However, Butterworth et al ${ }^{[25]}$ reported no change in MPC.

As far is Mean Platelet Count is concerned, such a significant difference was also found in comparison between ischemic and hemorrhagic stroke. A P value of .025 was obtained upon performing $\mathrm{T}$ Test. Such a finding has not been documented in the reference material we used so far and could be a spurious association. Such a difference can be explained by the fact that the platelets undergo modification in size and shape whilst releasing factors that aid in hemostasis in an attempt to seal the site of hemorrhage. Further studies are yet to confirm our findings if they haven't already been similarly noted elsewhere.

Mean Platelet Volume was also raised, showing a significant value of 0.00 . This is consistent with the studies conducted by Butterworth Et al, ${ }^{[25]}$ Farahnaz Ghahremanfard et al ${ }^{[22]}$ and Parvaiz. A. Shah et al..$^{[21]}$ However, many studies have come up with no such significance; one such study was conducted by Waseem F Al-Tameemi et al. MPV points to increased platelet reactivity and subsequent aggregation.

Platelet Distribution Width is also shown to have a significant association with a value of 0.00 . Although Waseem F Al-Tameemi et $\mathrm{al}^{[20]}$ showed no such finding, Parvaiz. A. Shah et $\mathrm{al}^{[21]}$ reported raised PDW in stroke patients. PDW represents variability in platelet size and high numbers could indicate an attempt to produce fresh platelets to aid in coagulation.

Although most of the above findings can be attributed to an actual pathological basis, further studies must be done to confirm the above findings in actual clinical practice. With consistent results being already produced, in the future, platelet indices can aid as a good prognostic tool to gauge the extent severity and recurrence of a cerebrovascular accident.

\section{Conclusion}

This Study has shown significant difference in the values of MPV, MPC and PDW between patients with stroke and normal patients who had come to the hospital for routine master health check up. Furthermore, findings show a significant difference in MPC between patients with hemorrhagic stroke and ischemic stroke, a finding not documented in similar studies so far. Except for the significance in hemorrhagic stroke patients, all other findings have been consistent with most studies.

Platelet indices are valuable pointers that are yet to be explored in their entirety and still hold many unique features that haven't been fully unraveled yet. In the future, with more such studies, they may serve the purpose of being a good prognostic tool and a valuable guide.

\section{Acknowledgements}

I wish to acknowledge my Guides Dr. Muthuvel and Dr. Yogalakshmi for guiding me with this study. Their patience and knowledge of the subject combined with their meticulous eye for detail has made me perfect every aspect of carrying out a study. I also wish to thank my good friend Ms. Architha Ellapalayam from Wageningen University, Netherlands, for providing me statistical input and assistance, thereby playing an important role in the study.

\section{Reference:}

1. Laki K .Our ancient heritage in blood clotting and some of its consequences. Annals of the New York Academy of Sciences.1972; 202: 297-307.

2. Machlus KR; Thon JN; Italiano JE. Interpreting the developmental dance of the megakaryocyte: A review of the cellular and molecular processes mediating platelet formation”. British Journal of Haematology. 2014; 165 (2): 227-36.

3. Jain NC. A scanning electron microscopic study of platelets of certain animal species. Thrombosis et diathesis haemorrhagica.1975 33 (3): 501-7.

4. Michelson D, Platelet Structure. ln: James G White, Editors. Platelets. Elsevier 2013, p. 117-118

5. Matarrese P; Straface E; Palumbo G. Mitochondria regulate platelet metamorphosis induced by opsonized zymosan A- 
activation and long-term commitment to cell death. FEBS Journal.2009 276 (3): 845-56.

6. Jagroop IA, Tsiara S, Mikhailidis DP. Mean platelet volume as an indicator of platelet activation: methodological issues. Platelets. 2003; 14: 335-6.

7. Tsiara S, Elisaf M, Jagroop IA, et al. Platelets as predictors of vascular risk: Is there a practical index of platelet activity? Clinical and Applied Thrombosis/Hemostasis. 2003; 9: 177-90.

8. Jakubowski JA, Thomson CB, Vaillancourt R, et al. Arachidonic acid metabolism by platelets of differing size. British Journal of Haematology 1983; 53: 503-511.

9. Giovanetti T, Nascimento A. Platelet Indices: Laboratory and Clinical Applications. Revista Brasileira de Hematologia e Hemoterapia 2011 33(2):164-165

10. Pizzuli L, Yang A, Martin JF, Luderitz B. Changes in platelet size and count in unstable angina compared to stable angina and non-cardiac chest pain. European Heart Journal, 1998; 19: $80-84$

11. Enticknap JB, Gooding PG, Lansley TS. Platelet size and function in ischemic heart disease. Atherosclerosis Research 1969; 10: 41-49.

12. Jagroop IA, Mikhailidis DP. Mean platelet volume is an independent risk factor for myocardial infarction, but not for coronary artery disease. British Journal of Haematology 2003; 120: 166-71.

13. Dougherty JH, Levy DE, Weksler BB. Platelet activation in acute cerebral ischemia. Lancet 1997; 1: 821-824.

14. Toghi H, Suzuki H, Tamura K, Kimura B. Platelet volume, aggregation and ATP release in cerebral thrombosis. Stroke 1991; 22: 17-21
15. Hartona S. Experiences from a multicenter Stroke register: a preliminary report. Bulletein of World Health Organisation 1976;54:541-53.

16. Phillip Bath. Acute stroke. In: David Machin, Simon Day, Sylvan Green (Ed). Textbook of clinical Trials. Wiley 2006;179-180.

17. Richard S. Snell (2006). Clinical neuroanatomy, 6. ed. Lippincott Williams \& Wilkins, Philadelphia. pp. 478-485.

18. Konkle B A, Disorders of Platelets and Vessel Wall ln: Kasper, Fauci, Loscalzo Editors, Harrison's Principles of Internal Medicine (19th edition), McGraw Hill, p 725-732

19. Mdlley TO, Langhorne P, Elton RA, Steward C. Platelet size in stroke patients. Stroke 1995; 26: 995-99

20. Al-Tameemi W, Ameen MM. Significance of Platelet Indices in Patients with Acute Ischemic Stroke. The Iraqui Journal of Agricultural Sciences. 2012 vol 10 (4):383-389

21. Shah P, Mir R, Kamili M. Role of Mean Platelet Volume in Ischemic Stroke. JK science 2013 Volume 15 (3): 136-139

22. Ghahremanfard F, Asghari N, Ghorbani R. The relationship between mean platelet volume and severity of acute ischemic brain stroke. Neurosciences 2013; Vol. 18 (2): 147-151

23. Bath PM, Butterworth RJ. Platelet size: measurement, physiology and vascular disease. Blood Coagul Fibrinolysis. 1996;7(2):157-61.

24. Jackson SR, Carter JM. Platelet volume: laboratory measurement and clinical application. Blood Rev. 1993;7(2):104-13.

25. Butterworth RJ, Bath PM. The relationship between mean platelet volume, stroke subtype and clinical outcome. Platelets.1998;9(6):359-64

*Corresponding author:

Dr Anish S Bharatwaj, B2, Soundaryam Appartments, EB Road, Mogappair East, Chennai 37 INDIA

Email: anish.sb@rediffmail.com

Financial or other Competing Interests: None. 